\title{
Antioxidant activity is required for the protective effects of cyclophilin A against oxidative stress
}

\author{
KIYOON KIM*, IN KYUNG OH*, KYUNG SIK YOON, JOOHUN HA, \\ INSUG KANG and WONCHAE CHOE
}

\author{
Department of Biochemistry and Molecular Biology, \\ Medical Science and Engineering Research Center for Bioreaction to Reactive Oxygen Species, \\ Biomedical Science Institute, School of Medicine, Kyung Hee University, Seoul 130-701, Republic of Korea
}

Received May 3, 2014; Accepted January 7, 2015

DOI: $10.3892 / \mathrm{mmr} .2015 .3392$

\begin{abstract}
Cyclophilin (Cyp) belongs to a group of proteins that have peptidyl-prolyl cis-trans isomerase (PPIase) activity. CypA is the major cellular target for the immunosuppressive drug cyclosporin A and mediates its actions. Previous studies have demonstrated that CypA has diverse cellular functions and have suggested that CypA may function as an antioxidant. The present study investigated the antioxidant activity of CypA and its association with PPIase activity. The purified CypA/wild-type (WT) and CypA/P16S mutant proteins were active in PPIase assays. A total antioxidant capacity assay revealed that the purified CypA/WT protein had significantly higher antioxidant activity, whereas the CypA/P16S mutant was defective in its antioxidant activity. To confirm the importance of CypA antioxidant activity, CypA/P16S was overexpressed in Chang human liver cells and the rate of cell death was measured following treatment with cisplatin or $\mathrm{H}_{2} \mathrm{O}_{2}$. Overexpression of CypA/WT protected the cells against cisplatin or $\mathrm{H}_{2} \mathrm{O}_{2}$-induced oxidative damage, however, the CypA/P16S mutant had no effect. These findings suggested that CypA exhibits a protective antioxidant effect.
\end{abstract}

\section{Introduction}

Cyclophilin (Cyp) is present in prokaryotes and eukaryotes and is an immunophilin and a cytosolic receptor for the immunosuppressive drug, cyclosporin A (1). In addition,

Correspondence to: Professor Wonchae Choe, Department of Biochemistry and Molecular Biology, Medical Science and Engineering Research Center for Bioreaction to Reactive Oxygen Species, Biomedical Science Institute, School of Medicine, Kyung Hee University, 1 Hoegi-dong, Dongdaemoon-gu, Seoul 130-701, Republic of Korea

E-mail:wchoe@khu.ac.kr

${ }^{*}$ Contributed equally

Key words: cyclophilin A, oxidative stress, peptidyl-prolyl cis-trans isomerase activity, antioxidant
Cyp possesses enzymatic peptidyl-prolyl cis-trans isomerase (PPIase) activity, which is essential for protein folding in vivo (2). PPIase activity has been suggested to facilitate protein folding, intracellular trafficking and the maintenance of multiprotein complex stability (3). There are several isoforms of Cyp, including CypA (4), CypB (5), СypC (6) and CypD (7). CypA is predominantly localized in the cytoplasm and is a highly conserved protein in mammalian cells $(4,8)$. In our previous study (9), CypA protected cells against cellular stresses, including hypoxia and cisplatin-induced effects, and it was hypothesized that the protective effects of CypA were, at least in part due, to its antioxidant activity (9). However, the antioxidant activity of CypA remains to be fully elucidated.

Several mutations in CypA have been identified. The CypA mutant, which carries a serine (Ser/S) instead of proline (Pro/P) at amino acid 16, has been reported to alter the folding/assembly pathway and its refolding intermediates have been observed to fall into a kinetic trap in the refolding process under the observed time course, resulting in a small fraction of multimerized CypA (10). It has been demonstrated that another CypA mutant, which carries alanine (Ala/A) instead of arginine $(\mathrm{Arg} / \mathrm{R})$ at amino acid 55, retains $<1 \%$ PPIase catalytic activity compared with the CypA/wild-type (WT) $(11,12)$.

The present study aimed to demonstrate the antioxidant activity of CypA by measuring the total antioxidant capacity of the purified CypA proteins. The association between the antioxidant activity of CypA and its PPIase catalytic activity was also investigated.

\section{Materials and methods}

Construction of glutatione S-transferase (GST)-CypA fusion proteins. The present study was approved by the Ethics Committee of Kyunghee University (Seoul, South Korea). Plasmids encoding GST fusion proteins were constructed using pGEX-KG vectors (American Type Culture Collection, Manassas, MA, USA). All the plasmid DNA was prepared using a modified alkaline lysis method (10). Briefly, the mutagens were created through site-direct mutagenesis as previously described (13) CypA/WT, CypA/R55A, the PPIase-defective mutate gene, and CypA/P16S, which is a mutant gene with a more flexible structure, were digested using BamHI and 
EcoRI [New England Biolab (NEB), Inc., Ipswich, MA, USA] and ligated into the pGEX-KG expression vector. The ligation mixture (NEB, Inc.) was used to transform an Escherichia coli DH5 $\alpha$ strain (Life Technologies, Grand Island, NY, USA). All the constructs were verified by DNA sequencing.

Site-directed mutagenesis of CypA. Mutations in CypA were produced by site-directed mutagenesis using modified rapid polymerase chain reaction (PCR) (14). Substitution of Ser16 into Pro and Ala55 into Arg were performed by PCR in two steps with a MyCycler (Bio-Rad, Hercules, CA, USA). Firstly, the two fragments, containing the sequences upstream and downstream of the Ser16 and Ala55 residues, were amplified using primers containing the desired mutations. A PCR mixture, total volume $50 \mu 1$ [2 $\mu \mathrm{g}$ CypA/WT template DNA; $1 \mu \mathrm{l}$ each forward and reverse primers (100 pmol; Macrogen, Inc., Seoul, Republic of Korea); $5 \mu 1$ 10X buffer; $3 \mu \mathrm{l}$ deoxyribonucleotide; $1 \mu \mathrm{l}$ taq polymerase (Takara Bio, Inc., Otsu, Japan); and $37 \mu 1$ distilled water], was used. The cycling conditions were as follows: $94^{\circ} \mathrm{C}$ for $5 \mathrm{~min}$, followed by 25 cycles of $94^{\circ} \mathrm{C}$ for $1 \mathrm{~min}, 55^{\circ} \mathrm{C}$ for $1 \mathrm{~min}$, and $72^{\circ} \mathrm{C}$ for $1 \mathrm{~min}$, the reaction was terminated by $72^{\circ} \mathrm{C}$ for $10 \mathrm{~min}$. The primer sequences used were as follows: R55A forward, AAATTTGGATCCATGGTCAACCCCACCG and reverse, GGCGGAATTCTTAGAGTTGTCCACAGTC; and P16S forward, ACTGTAAGCTTATGGTCAACCCCACCG and reverse, CCCGGGGATATCTTAGAGTTGTCCACAG. Secondly, the two amplified fragments were used as templates for a second PCR reaction at the same conditions, in which only a primer for the $5^{\prime}$ end of the first fragment and a primer for the 3 ' end of the second fragment were used, resulting in a full length Pro16 and Arg55 mutated CypA.

Expression and purification of the recombinant fusion proteins. The pGEX-KG, pGEX-KG/CypA/WT, pGEX-KG/CypA/P16S and pGEX-KG/CypA/R55A plasmids were transformed into the DH5 $\alpha$ E. coli strain. The bacterial cells were grown at $37^{\circ} \mathrm{C}$ in $200 \mathrm{ml}$ Lysogeny broth (Life Technologies) containing ampicillin $(100 \mu \mathrm{g} / \mathrm{ml}$; Duchefa Biochemie, Haarlem, Netherlands) from an overnight culture. At at absorbance ${ }_{600}$ of $\sim 0.6$ (NovaspecII; Biotek Instruments, Inc., Winooski, VT, USA), the bacterial cells were induced using $0.1 \mathrm{mM}$ isopropyl $\beta$-d-thiogalactopy ranoside (IPTG) for $3 \mathrm{~h}$ at $30^{\circ} \mathrm{C}$. The cells were harvested by centrifugation at $890 \mathrm{x}$ g for $15 \mathrm{~min}$ and resuspended in $2 \mathrm{ml} 1 \mathrm{X}$ phosphate-buffered saline (PBS; Bioworld, Bundang, South Korea) containing 1\% Triton X-100 (Sigma-Aldrich, St. Louis, MO, USA). The cells were maintained on ice and lyzed by sonication (Sonicator W-375 Cell disruptor; Heat Systems Ultrasonics Inc., Wehnrath, Germany). Following centrifugation at $890 \mathrm{xg}$ for $15 \mathrm{~min}$ at $4^{\circ} \mathrm{C}$, the cell debris was removed and the supernatant was incubated overnight with washed glutathione (GSH)-agarose (Sigma-Aldrich) at $4^{\circ} \mathrm{C}$ with gentle rotation (Rotamix SLRM I; Seoulin Bioscience Co., Seoul, South Korea). The samples were centrifuged at $100 \mathrm{x}$ g for $1 \mathrm{~min}$ at $4^{\circ} \mathrm{C}$ and the GST-only or the GST-CypA fusions bound to the GSH-agarose beads (Sigma-Aldrich) were washed five times with ice-cold PBS containing $1 \%$ Triton X-100. The target fusion protein was eluted from the resin using an elution buffer, containing $20 \mathrm{mM}$ reduced GSH in $100 \mathrm{mM}$ Tris- $\mathrm{HCl}$ (pH 9.0; Duchefa Biochemie) overnight at $4^{\circ} \mathrm{C}$. Following centrifugation at $100 \mathrm{x}$ g for $1 \mathrm{~min}$ at $4^{\circ} \mathrm{C}$, the supernatant containing the fusion proteins was mixed with 6X SDS sample buffer (Sigma-Aldrich), containing 0.35 M Tris-HCl (pH 6.8), 10.3\% SDS, $36 \%$ glycerol, $0.6 \%$ bromophenol blue and $0.6 \mathrm{M}$ dithiothreitol. The samples were analyzed by SDS-PAGE, native-PAGE and immunoblot analysis.

Removal of the GST by selective cleavage using thrombin. The GST tag was cleaved from the GST-CypA using a Thrombin CleanCleave ${ }^{\mathrm{TM}}$ kit (Sigma-Aldrich) according to the manufacturer's instructions. The fusion protein $(1 \mathrm{mg} / \mathrm{ml})$ in cleavage buffer containing $500 \mathrm{mM}$ Tris- $\mathrm{HCl}(\mathrm{pH}$ 8.0) and $100 \mathrm{mM} \mathrm{CaCl}_{2}$ (Duchefa Biochemie), was incubated at $4^{\circ} \mathrm{C}$ with gentle rotation overnight in the presence of $100 \mu \mathrm{l}$ bovine thrombin agarose beads (Sigma-Aldrich). Following the reaction, the thrombin agarose was removed from the mixture by centrifugation at $100 \mathrm{x}$ g for $1 \mathrm{~min}$. The supernatant was assessed by SDS-PAGE, native-PAGE and immunoblot analysis.

SDS-PAGE, immunoblot analysis and native-PAGE. The total cell lysate, purified fusion proteins and cleaved fusion proteins were separated by $12 \%$ SDS-PAGE (Life Technologies) and the proteins were transferred onto nitrocellulose membranes (Pall Corp., Pensacola, FL, USA). Transfer of the proteins was assessed by Ponceau red staining (Sigma-Aldrich) and the membranes were subsequently blocked for $1 \mathrm{~h}$ at room temperature in 3\% bovine serum albumin (Sigma-Aldrich) in Tris-buffered saline containing $10 \mathrm{mM}$ Tris- $\mathrm{HCl}(\mathrm{pH} 8.0$; Life Technologies) and $150 \mathrm{mM} \mathrm{NaCl}$ (Duchefa Biochemie), supplemented with $0.05 \%$ Tween-20 (TBST). The nitrocellulose membrane was washed with $1 \mathrm{X}$ TBST three times for $15 \mathrm{~min}$ and incubated with the following primary antibodies: Mouse monoclonal anti-GST (1:1,000; sc-138; Santa Cruz Biotechnology, Inc. Dallas, TX, USA), rabbit polyclonal anti-CypA (1:1,000; 07-313; Millipore, Billerica, MA, USA), mouse monoclonal anti-HA (1:1,000; sc-7392; Santa Cruz Biotechnology, Inc.) and mouse polyclonal antiGAPDH (1:1,000; csa-335; Enzo Life Sciences, Farmingdale, NY, USA), for $1 \mathrm{~h}$ at room temperature. Following washing with TBST three times for $15 \mathrm{~min}$, the membrane was incubated with horseradish peroxidase-conjugated secondary antibody for $45 \mathrm{~min}$ at room temperature.

Native-PAGE analysis was performed using a $12 \%$ gel. The stacking gel, separating gel and the running buffer were prepared as for SDS-PAGE, however, no SDS was added and the sample was not heated.

PPIase activity assay. The PPIase activity assay was performed, as described previously (15-17), with the suggested substrate solvent application (18). This assay determines the rate of conversion of cis-to-trans in proline-containing peptides, based on the principle that $\alpha$-chymotrypsin cleaves the peptide only when it is in the trans conformation (18). The $\mathrm{N}$-succinyl-Ala-Ala-Pro-phenylalanine- $p$-nitroanilide peptide substrate (Sigma-Aldrich) was dissolved in $>99 \%$ trifluoroethanol (Sigma-Aldrich) with $470 \mathrm{mM} \mathrm{LiCl}$ (Sigma-Aldrich) to prepare a $100 \mathrm{mM}$ stock solution, which was further diluted to $4 \mathrm{mM}$ prior to use. $\alpha$-chymotrypsin was dissolved 
A

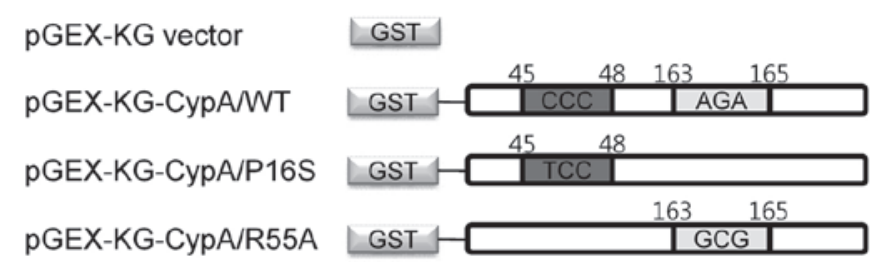

B

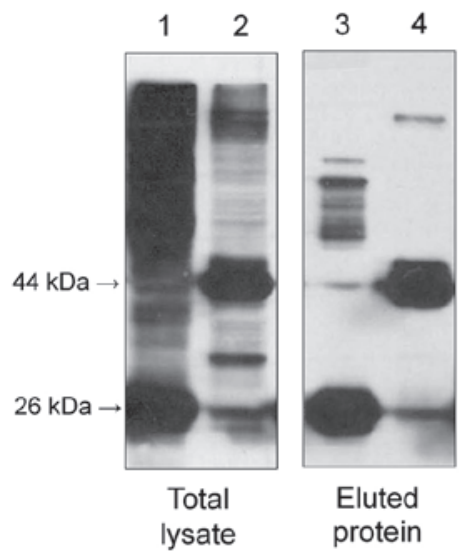

C

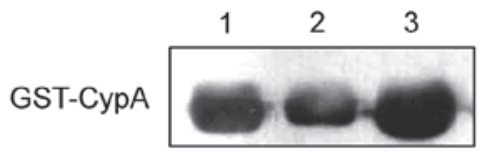

Figure 1. (A) Schematic of the GST-CypA constructs. (B) Western blot analysis of the purified GST-CypA fusion protein. The total GST protein lysate and the eluted GST protein lysate were separated by SDS-PAGE. The proteins were detected using an anti-GST antibody. Lane 1, total GST protein lysate; lane 2, total GST-CypA fusion protein lysate; lane 3, eluted GST protein lysate; Lane 4, eluted GST-CypA fusion protein lysate. (C) Western blot analysis of the purified GST-CypA/WT, GST-CypA/P16S, and GST-CypA/R55A fusion proteins. The proteins were detected using an anti-CypA antibody. Lane 1, eluted GST-CypA/WT; lane 2, eluted GST-CypA/P16S; lane 3, eluted GST-CypA/R55A. GST, glutatione S-transferase; CypA, cyclophilin A.

in $1 \mathrm{mM} \mathrm{HCl}$ (Junsei Chemical Co., Ltd, Tokyo, Japan) with $2 \mathrm{mM} \mathrm{CaCl}{ }_{2}$ to prpare a $1 \mathrm{M}$ stock solution. GST-CypA was diluted into $50 \mathrm{mM}$ HEPES (Sigma-Aldrich) and $86 \mathrm{mM} \mathrm{NaCl}(\mathrm{pH} 8.0)$, to prepare the PPIase buffer. In a $1 \mathrm{ml}$ cuvette (Sigma-Aldrich), $100 \mu 1$ protein $(10 \mu \mathrm{M}$ final concentration) was added to $890 \mu 1$ PPIase buffer. The reaction was initiated by the addition of $10 \mu 1(40 \mu \mathrm{M})$ peptide substrate and $\alpha$-chymotrypsin (Sigma-Aldrich) of $0.5 \mu \mathrm{l}(500 \mu \mathrm{M})$, followed by rapid mixing using a pipette. The change in absorbance at $390 \mathrm{~nm}$, following the cleavage of the trans form of the peptide and release of $p$-nitroaniline, was monitored using a biosciences spectrophotometer (VICTOR; PerkinElmer, Waltham, MA, USA).

Antioxidant activity assay. The antioxidant activity was determined using a Total Antioxidant Capacity Assay kit (BioVision Research Products, Mountain View, CA, USA) and measured by monitoring the reduction of $\mathrm{Cu}^{++}$reagent by the increase of absorbance at $570 \mathrm{~nm}$. The degree of quenching of radical generation in individual samples, indicative of the presence of antioxidant activity, was quantified by comparison with a traditional standard trolox (Sigma-Aldrich) and the assay results were expressed in terms of $\mu \mathrm{mol} /$ trolox.

Cell culture and reagent. Chang human liver cells (American Type Culture Collection) were cultured in Dulbecco's modified Eagle's medium (GE Healthcare, Logan, WV, USA) supplemented with $10 \%$ (v/v) fetal bovine serum (FBS; GE Healthcare) and antibiotics [100 U/ml penicillin (Duchefa Biochemie) and $100 \mu \mathrm{g} / \mathrm{ml}$ streptomycin sulfate (Sigma-Aldrich)] in a $5 \% \mathrm{CO}_{2}$ incubator. To induce oxidative stress, the cells were treated with cisplatin $(0,15$ or $20 \mu \mathrm{mol} / \mathrm{l})$ or $\mathrm{H}_{2} \mathrm{O}_{2}(0$, 400 or $500 \mu \mathrm{mol} / \mathrm{l}$ ) for $24 \mathrm{~h}$.

Transfection. CypA/WT and CypA/R55A were tagged (hemagglutinin tag, 5'-TAC CCA TAC GAC GTC CCA GAC TAC GCT- 3 ') at the 5 ' end. The cells were cultured in a 12-well plate at $37^{\circ} \mathrm{C}$ in a $5 \% \mathrm{CO}_{2}$ incubator for a 3-(4,5-dimethylthiazol-2-yl)-2,5-diphenyltetrazolium bromide (MTT) assay. The cells were transfected using Lipofectamine $2000^{\mathrm{TM}}$ reagent (Invitrogen Life Technologies, Carlsbad, CA, USA), according to the manufacturer's instructions. Following transfection, cells were also incubated in the same conditions for 18-24 h.

MTT assay. Following $24 \mathrm{~h}$ treatment with cisplatin or $\mathrm{H}_{2} \mathrm{O}_{2}$, the cell viability was evaluated using an MTT conversion assay in a 12-well plate. The culture medium (DMEM supplementd with $10 \% \mathrm{FBS}, 100 \mathrm{U} / \mathrm{ml}$ penicillin and $100 \mu \mathrm{g} / \mathrm{ml}$ streptomycin sulfate) was replaced with $1 \mathrm{ml}$ medium containing $0.5 \mathrm{mg} / \mathrm{ml}$ MTT (Sigma-Aldrich; dissolved in filtration water) and incubated for $60 \mathrm{~min}$ at $37^{\circ} \mathrm{C}$. The medium was then carefully aspirated from the plates and the blue-colored tetrazolium crystals, resulting from mitochondrial enzymatic activity, on the MTT substrate were solubilized in $150 \mu \mathrm{l}$ $100 \%$ dimethylsulfoxide (Junsei, Tokyo, Japan). The absorbance was measured at $595 \mathrm{~nm}$ in a Model 680 Microplate 
A

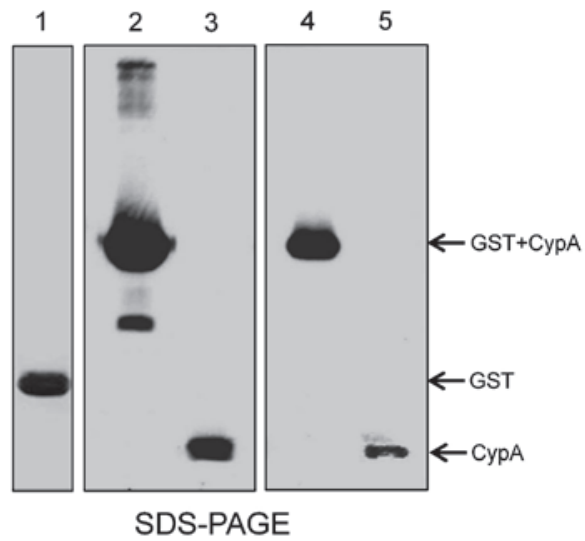

B

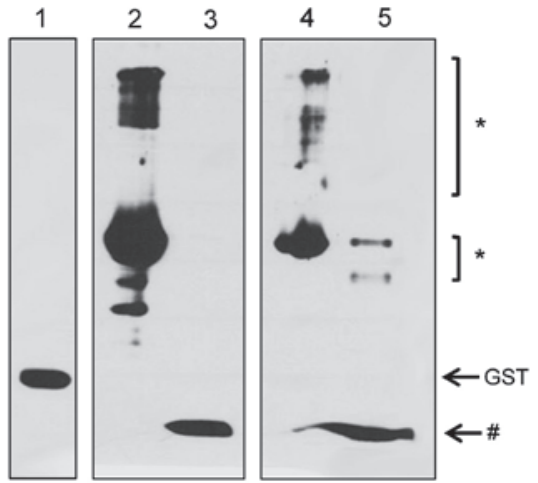

Native-PAGE

Figure 2. (A) Western blot analysis of the recombinant CypA proteins. CypA recombinant fusion proteins were separated by SDS-PAGE following GST cleavage. The proteins were detected using an anti-GST antibody (lane 1) and an anti-CypA antibody (lanes 2-5). Lane 1, eluted GST protein; lane 2, eluted GST-CypA/WT protein; lane 3, CypA/WT protein following cleavage; lane 4, eluted GST-CypA/P16S protein; lane 5, recombinant CypA/P16S protein following GST cleavage. (B) Recombinant CypA fusion proteins were separated by Native-PAGE gel. The proteins were detected using anti-GST (lane 1) and anti-CypA (lane 2-5). Lane 1, eluted GST protein alone; lane 2, eluted GST-CypA/WT protein; lane 3, CypA/WT protein following cleavage; lane 4, eluted GST-CypA/P16S protein; lane 5, CypA/P16S protein following cleavage. "Multimers of CypA protein; " monomer of CypA protein. CypA, cyclophilin; GST, glutatione S-transferase.

A

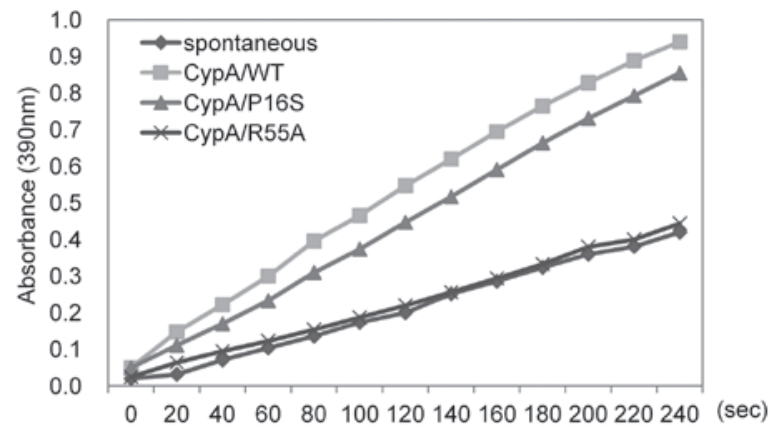

B

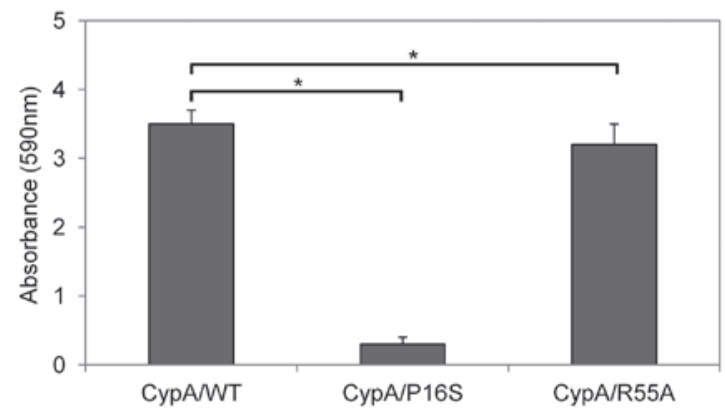

Figure 3. (A) PPIase activity of $10 \mu \mathrm{M}$ GST-CypA WT, GST-CypA/P16S, GST-CypA/R55A and without enzymes. (B) Antioxidant catalytic activity of $1 \mu \mathrm{M}$ CypA WT, CypA/P16S and CypA/R55A protein. The data are expressed as the mean \pm standard deviation of three independent experiments. ${ }^{*} \mathrm{P}<0.05$ vs. Cyp/WT-transfected cells. CypA, cyclophilin A; GST, glutatione S-transferase; WT, wild-type; PPIase, peptidyl-prolyl cis-trans isomerase. 
A

\author{
pcDNA3-CурAMT \\ pcDNA3-CypA/P16S
}

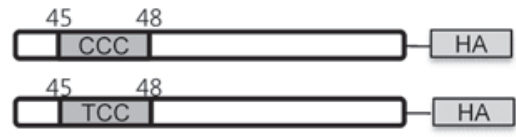

B

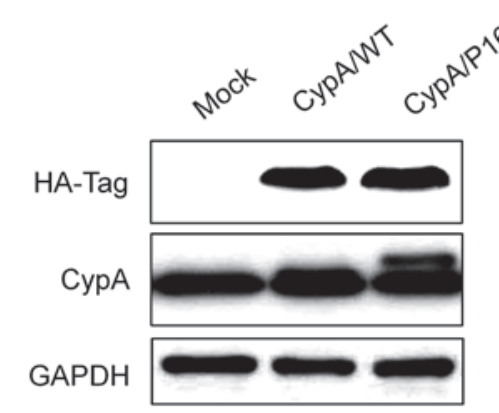

C

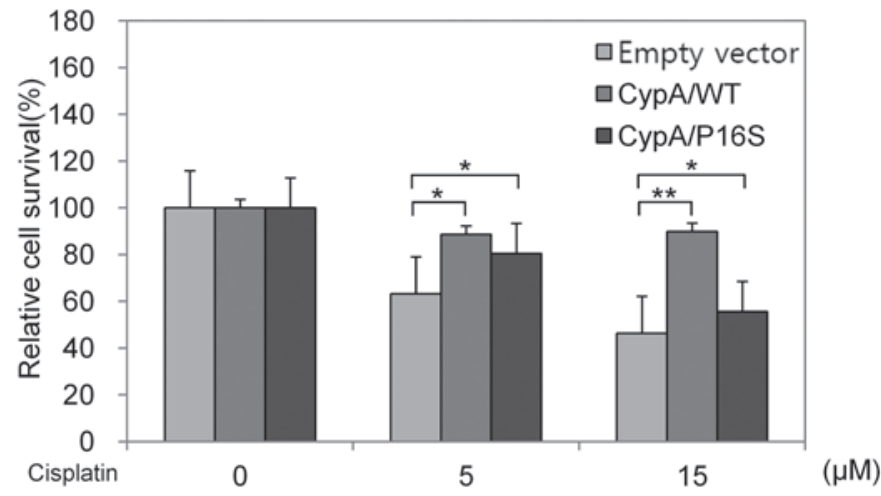

D

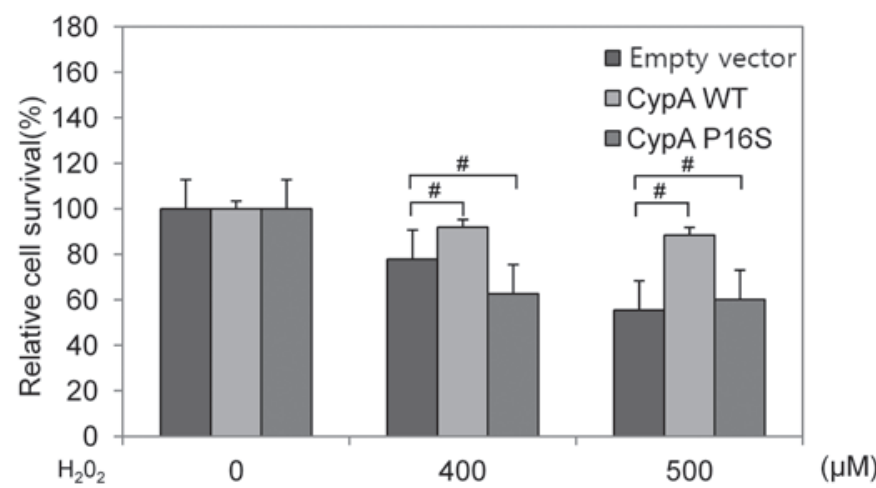

Figure 4. Effects of CypA on cell death following treatment with cisplatin or $\mathrm{H}_{2} \mathrm{O}_{2}$. (A) Schematic of the CypA constructs in mammalian expression vectors. (B) Protein expression level of CypA. The expression of CypA was determined by western blotting using anti-CypA or anti-HA antibodies. Chang cells were transfected with the empty vector, CypA/WT and CypA/P16S and were treated with (C) 0,5 or $15 \mu \mathrm{M}$ cisplatin or (D) 0,400 or $500 \mu \mathrm{M} \mathrm{H}_{2} \mathrm{O}_{2}$ for $24 \mathrm{~h}$. Cell viability was measured using an MTT assay and survival rate was calculated relative to that of $0 \mu \mathrm{M} \mathrm{H}_{2} \mathrm{O}_{2}$. The data are expressed as the mean \pm standard deviation of three independent experiments. ${ }^{*} \mathrm{P}<0.05$ and ${ }^{* *} \mathrm{P}<0.01$ vs. empty vector-transfected cells treated with cisplatin, ${ }^{*} \mathrm{P}<0.05$ vs. empty vector-transfected cells treated with $\mathrm{H}_{2} \mathrm{O}_{2}$. WT, wild-type; $\mathrm{HA}$, hemagglutinin; Cyp, cyclophilin.

Reader (Bio-Rad Laboratories, Inc., Hercules, CA, USA). Cell survival was expressed as the percentage of absorbance relative to that of the untreated cells.

\section{Results}

Purification of the expressed GST-CypA fusion proteins. In order to investigate the biochemical activity of CypA, the GST-CypA/WT, GST-CypA/P16S and GST-CypA/R55A plasmids were expressed in E. coli. A schematic of the constructed expression plasmids is shown in Fig. 1A. The recombinant GST-CypA fusion proteins were successfully overexpressed by the addition of $0.1 \mathrm{mM}$ IPTG in E. coli (Fig. 1B). The
GST-only and GST-CypA fusion proteins were eluted using GSH-beads (Fig. 1B). The expression levels of GST-CypA/WT, GST-CypA/P16S and GST-CypA/R55A were analyzed by western blotting (Fig. 1C). The $18-\mathrm{kDa}$ recombinant CypA proteins were purified following GST cleavage with thrombin, and the purified proteins were confirmed by western blotting using an anti-CypA antibody (Fig. 2A). A small fraction of the CypA/P16S mutant protein has been reported to exhibit a multimerized structure due to its low yield of refolding (10). Therefore, in order to confirm the multimerized CypA/P16S, native-PAGE in non-denaturing conditions was used (Fig. 2B). The results demonstrated that a significant level of multimerized complexes of CypA/P16S remains, consistent with 
the previous report (10). However, CypA/WT remained as a monomer (Fig. 2A).

PPIase and antioxidant activity assays of purified CypA protein. The PPIase activity of the purified CypA protein was measured. The PPIase activity assay is based on the observation that $\alpha$-chymotrypsin cleaves the $C$-terminal amide bond only in the trans X-pro conformer of the chromogenic substrate, X-Pro-Phe-pNA. The rapid hydrolysis perturbs the cis-trans conformational equilibrium, which enables the PPIase-catalyzed cis-to-trans isomeriztion to be monitored. The PPIase activity was assayed in an $\alpha$-chymotrysin coupled assay. As shown in Fig. 3A, recombinant CypA/WT and CypA/P16S exhibited PPIase activity, although CypA/P16S demonstrated marginally less efficient activity. Consistent with a previous report, the CypA/R55A was defective in its PPIase activity, as shown in Fig. 3A (13). The total antioxidant activity of the purified CypA protein was also measured. CypA/WT and CypA/R55A demonstrated significantly higher antioxidant activities, however, CypA/P16S exhibited no antioxidant activity (Fig. 3B). GST-only was used as a negative control.

Overexpression of CypA reduces cell death induced by cisplatin or $\mathrm{H}_{2} \mathrm{O}_{2}$. To confirm the importance of the antioxidant activity of CypA during oxidative stress, HA-tagged CypA was expressed in Chang human liver cells (Fig. 4A). The overexpression of CypA/WT or CypA/P16S was analyzed by western blotting (Fig. 4B). The effects of overexpressed CypA on reactive oxygen species-mediated cell death was determined using an MTT assay with various concentrations of $\mathrm{H}_{2} \mathrm{O}_{2}(400$ and $500 \mu \mathrm{M})$ for $24 \mathrm{~h}$. The CypA/WT-transfected cells demonstrated a higher survival rate compared with the empty vector-transfected cells. By contrast, the CypA/P16S-transfected cells exhibited a higher sensitivity to $\mathrm{H}_{2} \mathrm{O}_{2}$-mediated cell death compared with the CypA/WT-transfected cells (Fig. 4C). The chemoresistance of the CypA/WT- and CypA/P16S-transfected cells following treatment with cisplatin were monitored using an MTT assay. Cisplatin is known to induce apoptosis, partly through the generation of oxidative stress (19-21). The cells transfected with CypA/WT had a reduced level of cell death following treatment with cisplatin, compared with the empty vector-transfected cells. By contrast, the CypA/P16S-transfected cells exhibited a lower survival rate following treatment with cisplatin, compared with the CypA/WT-transfected cells (Fig. 4D). These findings suggested that antioxidant activity is required for the protective effects of CypA against oxidative stress.

\section{Discussion}

CypA protects cells from several types of cellular stress, including oxidative stress (14) and endoplasmic reticulum stress (22). PPIase activity is reported to be associated with several cellular functions, in addition to its biochemical activity (23). Several reports have demonstrated that the PPIase activity of CypA is important (24) and our previous study revealed that overexpression of CypA protects several cell lines from oxidative stress in a PPIase activity-dependent manner (9). However, the antioxidant activity of CypA and its importance remain to be fully elucidated. Therefore, the present study focused on the antioxidant activity of CypA and identified for the first time, to the best of our knowledge, its importance in the cellular response to oxidative stress.

The antioxidant activity of recombinant CypA, purified from bacterial extracts, was measured using a Total Antioxidant Capacity Assay kit. The results demonstrated that a significant level of antioxidant activity was associated with CypA/WT and CypA/R55A, while CypA/P16S was defective in antioxidant activity. The PPIase activity of each protein was also measured and its association with antioxidant activity was investigated. As shown in Fig. 2A, CypA/WT and CypA/P16S exhibited PPIase activity, although CypA/P16S had marginally less efficient activity. This indicated that Pro16 in the structure of CypA does not affect the catalytic PPIase activity since Pro16 is located at a region distant from the catalytic center of the PPIase. In addition, these results suggested that the antioxidant activity of CypA is independent of PPIase activity, since CypA/R55A, which is defective in PPIase activity, exhibits antioxidant activity. Notably, CypA/P16S formed a multimerized complex, while CypA/WT existed as a monomer, as shown in Fig. 2B. Multimerization may inhibit the antioxidant activity of CypA/P16S. However, further studies are required to elucidate the association between the multimerization of CypA and its antioxidant activity.

Our previous study demonstrated that the overexpression of CypA/WT may be important in tumorigenesis by reducing apoptosis under hypoxic conditions and by treatment with cisplatin (9). Therefore, the present study hypothesized that the overexpression of CypA/WT, however, not CypA/P16S, may reveal a protective effect on oxidative stress-induced cell death. As hypothesized, the CypA/WT protected the cells from $\mathrm{H}_{2} \mathrm{O}_{2}$ or cisplatin-mediated cell death, while the CypA/P16S mutant was unable to protect the cells (Fig. 4).

In conclusion, the present study demonstrated that antioxidant activity was associated with CypA and was independent of PPIase activity. In addition, the antioxidant activity of CypA was required for the protective effects of CypA against $\mathrm{H}_{2} \mathrm{O}_{2}$ or cisplatin-mediated cell death. These findings may be useful for identifying a novel chemotherapeutic target in tumor cells, since cancer cells are usually resistant to oxidative stress, which is induced by anti-cancer drugs, such as Cisplatin (25).

\section{Acknowledgements}

This study was supported by grants from Kyung Hee University (Seoul, Republic of Korea) in 2012 (no. KHU-20121733) and the Basic Science Research Program through the National Research Foundation of Korea, funded by the Ministry of Education (no. NRF-2013R1A1A2060694).

\section{References}

1. Handschumacher RE, Harding MW, Rice J, Drugge RJ and Speicher DW: Cyclophilin: a specific cytosolic binding protein for cyclosporin A. Science 226: 544-547, 1984.

2. Caroni P, Rothenfluh A, McGlynn E and Schneider C: $\mathrm{S}$-cyclophilin. New member of the cyclophilin family associated with the secretory pathway. J Biol Chem 266: 10739-10742, 1991.

3. Andreeva L, Heads R and Green CJ: Cyclophilins and their possible role in the stress response. Int J Exp Pathol 80: 305-315, 1999.

4. Wang P and Heitman J: The cyclophilins. Genome Biol 6: 226, 2005 . 
5. Price ER, Zydowsky LD, Jin MJ, Baker CH, McKeon FD and Walsh CT: Human cyclophilin B: a second cyclophilin gene encodes a peptidyl-prolyl isomerase with a signal sequence. Proc Natl Acad Sci USA 88: 1903-1907, 1991.

6. Schneider H, Charara N, Schmitz R, et al: Human cyclophilin C: primary structure, tissue distribution, and determination of binding specificity for cyclosporins. Biochemistry 33: 8218-8224, 1994.

7. Carpentier M, Allain F, Haendler B, et al: Two distinct regions of cyclophilin B are involved in the recognition of a functional receptor and of glycosaminoglycans on T lymphocytes. J Biol Chem 274: 10990-10998, 1999.

8. Harding MW, Handschumacher RE and Speicher DW: Isolation and amino acid sequence of cyclophilin. J Biol Chem 261: 8547-8555, 1986.

9. Choi KJ, Piao YJ, Lim MJ, et al: Overexpressed cyclophilin A in cancer cells renders resistance to hypoxia- and cisplatin-induced cell death. Cancer Res 67: 3654-3662, 2007.

10. Xu LR, Yan X, Luo M, Guan YX and Yao SJ: Preparation, characterization and refolding in vitro of a recombinant human cyclophilin A mutant: effect of a single Pro/Ser substitution on cyclophilin A structure and properties. Biotechnol Prog 24: 302-310, 2008

11. Helekar SA and Patrick J: Peptidyl prolyl cis-trans isomerase activity of cyclophilin A in functional homo-oligomeric receptor expression. Proc Natl Acad Sci USA 94: 5432-5437, 1997.

12. Morita T, Kawabata T, Horikawa N, et al: Therapeutic consideration of patients with mental diseases. Seishin Shinkeigaku Zasshi 94: 1092-1098, 1992 (In Japanese).

13. Zydowsky LD, Etzkorn FA, Chang HY, et al: Active site mutants of human cyclophilin A separate peptidyl-prolyl isomerase activity from cyclosporin A binding and calcineurin inhibition. Protein Sci 1: 1092-1099, 1992.

14. Gavin PD, Devenish RJ and Prescott M: FRET reveals changes in the F1-stator stalk interaction during activity of F1F0-ATP synthase. Biochim Biophys Acta 1607: 167-179, 2003.
15. Fischer G, Bang H, Ludwig B, Mann K and Hacker J: Mip protein of Legionella pneumophila exhibits peptidyl-prolyl-cis/trans isomerase (PPlase) activity. Mol Microbiol 6: 1375-1383, 1992.

16. Rahfeld JU, Schierhorn A, Mann K and Fischer G: A novel peptidyl-prolyl cis/trans isomerase from Escherichia coli. FEBS Lett 343: 65-69, 1994.

17. Rouvière PE and Gross CA: SurA, a periplasmic protein with peptidyl-prolyl isomerase activity, participates in the assembly of outer membrane porins. Genes Dev 10: 3170-3182, 1996.

18. Kofron JL, Kuzmic P, Kishore V, Colon-Bonilla E and Rich DH: Determination of kinetic constants for peptidyl prolyl cis-trans isomerases by an improved spectrophotometric assay. Biochemistry 30: 6127-6134, 1991

19. Jiang Y, Guo C, Vasko MR and Kelley MR: Implications of apurinic/apyrimidinic endonuclease in reactive oxygen signaling response after cisplatin treatment of dorsal root ganglion neurons. Cancer Res 68: 6425-6434, 2008.

20. Santos NA, Catao CS, Martins NM, Curti C, Bianchi ML and Santos AC: Cisplatin-induced nephrotoxicity is associated with oxidative stress, redox state unbalance, impairment of energetic metabolism and apoptosis in rat kidney mitochondria. Arch Toxicol 81: 495-504, 2007.

21. Marullo R, Werner E, Degtyareva N, et al: Cisplatin induces a mitochondrial-ROS response that contributes to cytotoxicity depending on mitochondrial redox status and bioenergetic functions. PLoS One 8: e81162, 2013.

22. Kim J, Choi TG, Ding Y, et al: Overexpressed cyclophilin B suppresses apoptosis associated with $\mathrm{ROS}$ and $\mathrm{Ca}_{2}{ }^{+}$homeostasis after ER stress. J Cell Sci 121: 3636-3648, 2008.

23. Hunter T: Prolyl isomerases and nuclear function. Cell 92: 141-143, 1998.

24. Jin ZG, Melaragno MG, Liao DF, et al: Cyclophilin A is a secreted growth factor induced by oxidative stress. Circ Res 87: 789-796, 2000.

25. Pratibha R, Sameer R, Rataboli PV, Bhiwgade DA and Dhume CY: Enzymatic studies of cisplatin induced oxidative stress in hepatic tissue of rats. Eur J Pharmacol 532: 290-293, 2006. 